«Системні технологіï» 5 (136) 2021 «System technologies»

DOI 10.34185/1562-9945-5-136-2021-15

УДК 517.958:519.63

О.М. Алексєєв, М.Г. Бердник

\title{
МАТЕМАТИЧНА МОДЕЛЬ І МЕТОД РІШЕННЯ УЗАГАЛЬНЕНОЇ ЗАДАЧІ НЕЙМАНА ТЕПЛООБМІНУ ТІЛА ПІВСФЕРИЧНОЇ ФОРМИ
}

Анотація. У статті вперше побудована математична модель розрахунку температурних полів для півсферичного тіла у вигляді крайовоі задачі математичноі фізики для гіперболічного рівняння теплопровідності з граничними умовами Неймана, а також знайдено температурне поле у вигляді збіжних рядів.

Ключові слова: комплексний ряд Фур'є, крайова задача Неймана, інтегральне перетворення Лапласа, час релаксаціі.

Постановка проблеми. Найважливішим завданням у вирішенні проблеми оцінки ресурсного потенціалу території на тверді корисні копалини є підвищення геологічної і техніко-економічної ефективності бурових робіт, що неможливо без сучасного техніко-технологічного забезпечення цих робіт. Буріння свердловин є основним способом розвідки корисних копалин. При цьому найбільш ефективним в даний час і доступному в майбутньому способом буріння свердловин в твердих породах є алмазне буріння із застосуванням алмазного бурового долота. Алмазне бурове долото в процесі роботи сильно нагрівається за рахунок тепловиділення в зоні контакту і охолоджується потужним потоком бурового розчину. Врахування дії температурного чинника створює основу для прогнозування можливої величини механічної швидкості алмазного буріння, сприяє правильному вибору технологічних параметрів, об'єктивного контролю їх в процесі буріння, дозволяє намітити шляхи вдосконалення алмазного інструменту. Питання підвищення продуктивності, якості та економічності буріння шляхом створення, впровадження та вдосконалення алмазних породоруйнуючих інструментів мають постійну актуальність.

Аналіз останніх досліджень і публікацій. Аналіз праць [1-4] показав, що більшість результатів мають частковий характер, єдиний погляд на фізичну картину, що має місце при бурінні, відсутній, а відомі моделі не дають змогу прогнозувати температуру алмазного бурового долота з урахуванням кутової швидкості буріння та кінцевої швидкості поширення тепла.

(C) Алексєєв О.М., Бердник М.Г., 2021 


\section{«Системні технологіï» 5 (136) 2021 «System technologies»}

Мета статті. Для моделювання температурного поля слід наблизити форму долота до класичного і зробити ряд розумних спрощень. 3 огляду на незначні розміри бурових алмазів в порівнянні з розмірами матриці, i їх високу теплопровідність, будемо вважати, що вплив геометрії алмазів незначний.

Метою роботи є побудова нової математичної моделі розрахунку температурних полів для півсферичного тіла, яке наближено моделює роботу алмазного долота, у вигляді крайової задачі математичної фізики а також знаходження рішень отриманої крайової задачі.

Викладення основного матеріалу дослідження. Розглянемо розрахунок температурного поля тіла півсферичної форми у циліндричній системі координат $(r, \varphi, z)$ радіуса $\mathrm{R}$ з твірною лінією $r=\sqrt{R^{2}-(z-R)^{2}} \quad$ с центром в точці $\left(0, \frac{\pi}{2}, R\right)$ (рис. 1 ).

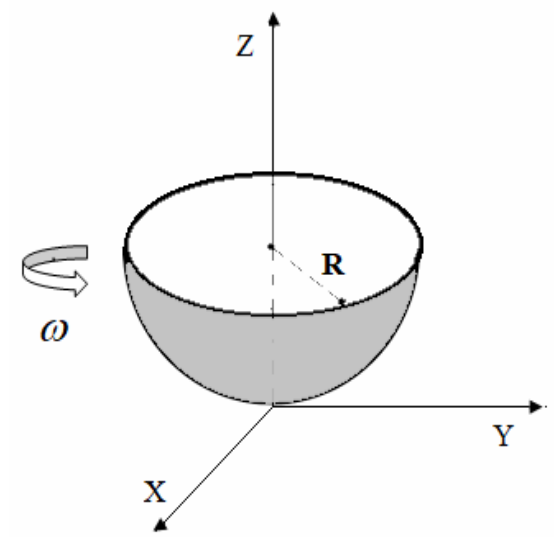

Рисунок 1 - Тіло півсферичної форми з твірною лінією $r=\sqrt{R^{2}-(z-R)^{2}}$

Тіло обертається навколо осі OZ з постійною кутовою швидкістю $\omega$, а швидкість поширення тепла $є$ відомою величиною. Теплофізичні властивості тіла не залежать від температури, а внутрішні джерела тепла відсутні.

У початковий момент часу температура тіла постійна $G_{0}$, а на бічній поверхні тіла відоме значення теплового потока $V(\varphi, z)$. При $\mathrm{z}=\mathrm{R}$ відоме значення теплового потока $G_{1}(r, \varphi)$.

В [5] отримано узагальнене рівняння переносу енергії для рушійного елемента суцільного середовища, з урахуванням скінченності величини швидкості поширення тепла. Згідно [5] узагальнене рівняння балансу енергії твердого тіла, яке обертається з постійною кутовою швидкістю $\omega$ навколо осі OZ, тепло- 


\section{«Системні технології» 5 (136) 2021 «System technologies»}

фізичні властивості якого не залежать від температури, а внутрішні джерела тепла відсутні, в циліндричній системи координат приймає вигляд:

$$
\gamma \mathrm{c}\left\{\frac{\partial \mathrm{T}}{\partial \mathrm{t}}+\omega \frac{\partial T}{\partial \varphi}+\tau_{r}\left[\frac{\partial^{2} T}{\partial t^{2}}+\omega \frac{\partial^{2} T}{\partial \varphi \partial t}\right]\right\}=\lambda\left[\frac{\partial^{2} T}{\partial r^{2}}+\frac{1}{r} \frac{\partial T}{\partial r}+\frac{1}{r^{2}} \frac{\partial^{2} T}{\partial \varphi^{2}}+\frac{\partial^{2} T}{\partial z^{2}}\right]
$$

де $\gamma$ - щільність середовища; с - питома теплоємність; $T(\rho, \varphi, z, t)$ температура середовища; $\lambda$ - коефіцієнт теплопровідності; $\mathrm{t}$-час; $\tau_{r}$ - час релаксації.

Математично задача визначення температурного поля тіла полягає в інтегруванні диференціального рівняння теплопровідності (1) в області, $D=\left\{(r, \varphi, z, t) \mid r \in\left(0, \sqrt{R-(z-R)^{2}}\right), \varphi \in(0,2 \pi), \mathrm{z} \in(0, \mathrm{R}), \mathrm{t} \in(0, \infty)\right\}$, що з урахуванням прийнятих допущень запишеться у вигляді:

$$
\frac{\partial \theta}{\partial t}+\omega \frac{\partial \theta}{\partial \varphi}+\tau_{r} \frac{\partial^{2} \theta}{\partial t^{2}}+\tau_{r} \omega \frac{\partial^{2} \theta}{\partial \varphi \partial t}=a\left[\frac{\partial^{2} \theta}{\partial r^{2}}+\frac{1}{r} \frac{\partial \theta}{\partial r}+\frac{1}{r^{2}} \frac{\partial^{2} \theta}{\partial \varphi^{2}}+\frac{\partial^{2} \theta}{\partial z^{2}}\right]
$$

3 початковими умовами

$$
\theta(r, \varphi, z, 0)=0, \quad \frac{\partial \theta(r, \varphi, z, 0)}{\partial t}=0
$$

і граничними умовами

$$
\left.\int_{0}^{t} \frac{\partial \theta}{\partial r}\right|_{r=\sqrt{R^{2}-(z-R)^{2}}} e^{\frac{r-t}{\tau_{r}}} d r=\left.G(\varphi, z) \quad \int_{0}^{t} \frac{\partial \theta}{\partial z}\right|_{z=R} e^{\frac{z-t}{\tau_{r}}} d z=\Lambda(r, \varphi)
$$

де $\theta=\frac{T(r, \varphi, z, t)-G_{0}}{T_{\max }-G_{0}}-$ відносна температура тіла; $G(\varphi, z)=\frac{V(\varphi, z) \tau_{r}}{\lambda\left(T_{\max }-G_{0}\right)}$; $\Lambda(r, \varphi)=\frac{G_{1}(r, \varphi) \tau_{r}}{\lambda\left(T_{\max }-G_{0}\right)} ; G(\varphi, z), \Lambda(r, \varphi) \in \mathrm{C}(0,2 \pi) ; a=\frac{\lambda}{c \gamma}$ - коефіцієнт температуропровідності.

Тоді рішення крайової задачі (2)-(4) $\theta(r, \varphi, z, t) \in$ двічі неперервно диференційованим за $r, \varphi, z$, один раз за $\mathrm{t}$ в області D i неперервним на $\bar{D}$ [6], тобто $\theta(r, \varphi, z, t) \in C^{2}(D) \cap C(\bar{D})$, а функції $G(\varphi, z), \Lambda(r, \varphi), \theta(r, \varphi, z, t)$, можуть бути розкладені в комплексний ряд Фур'є [6] 
«Системні технології» 5 (136) 2021 «System technologies»

$$
\begin{aligned}
& \left\{\begin{array}{c}
\theta(r, \varphi, z, t) \\
G(\varphi, z) \\
\Lambda(r, \varphi)
\end{array}\right\}=\sum_{n=-\infty}^{+\infty}\left\{\begin{array}{c}
\theta_{n}(r, z, t) \\
G_{n}(z) \\
\Lambda_{n}(r)
\end{array}\right\} \cdot \exp (\mathrm{i} n \varphi)
\end{aligned}
$$

3 огляду на те, що $\theta(r, \varphi, z, t)$ функція дійсна, надалі обмежимося розглядом $\theta_{n}(r, z, t)$ для $\mathrm{n}=0,1,2, \ldots$, тому що $\theta_{n}(r, z, t)$ і $\theta_{-n}(r, z, t)$ будуть комплексно спряженими [6]. Підставляючи значення функцій 3 (5) у (2)-(4), в результаті одержимо систему диференціальних рівнянь

$$
\frac{\partial \theta_{n}^{(i)}}{\partial t}+\vartheta_{n}^{(i)} \theta_{n}^{\left(m_{i}\right)}+\tau_{r} \frac{\partial^{2} \theta_{n}^{(i)}}{\partial t^{2}}+\tau_{r} \vartheta_{n}^{(i)} \frac{\partial \theta_{n}^{\left(m_{i}\right)}}{\partial t}=a\left[\frac{\partial^{2} \theta_{n}^{(i)}}{\partial r^{2}}+\frac{1}{r} \frac{\partial \theta_{n}^{(i)}}{\partial r}-\frac{n^{2}}{r^{2}} \theta_{n}^{(i)}+\frac{\partial^{2} \theta_{n}^{(i)}}{\partial z^{2}}\right]
$$

3 початковими умовами

$$
\theta_{n}^{(i)}(\rho, z, 0)=0, \quad \frac{\partial \theta_{n}^{(i)}(\rho, z, 0)}{\partial t}=0
$$

і граничними умовами

$$
\left.\int_{0}^{t} \frac{\partial \theta_{n}^{(i)}}{\partial r}\right|_{r=\sqrt{R^{2}-(z-R)^{2}}} e^{\frac{r-t}{\tau_{r}}} d r=\left.G_{n}^{(i)}(z) \quad \int_{0}^{t} \frac{\partial \theta_{n}^{(i)}}{\partial z}\right|_{z=R} e^{\frac{z-t}{\tau_{r}}} d z=\Lambda_{n}^{(i)}(r)
$$

Застосовуємо до системи диференціальних рівнянь (6) з умовами (7)-(8) інтегральне перетворення Лапласа [7]. У результаті одержуємо систему диференціальних рівнянь

$$
s \widetilde{\theta}_{n}^{(i)}+\vartheta_{n}^{(i)}\left(\widetilde{\theta}_{n}^{\left(m_{i}\right)}+\tau_{r} s \widetilde{\theta}_{n}^{\left(m_{i}\right)}\right)+\tau_{r} s^{2} \widetilde{\theta}_{n}^{(i)}=a\left[\frac{1}{r} \frac{\partial}{\partial r}\left(r \frac{\partial \widetilde{\theta}_{n}^{(i)}}{\partial r}\right)-\frac{n^{2}}{r^{2}} \widetilde{\theta}_{n}^{(i)}+\frac{\partial^{2} \widetilde{\theta}_{n}^{(i)}}{\partial z^{2}}\right]
$$

з граничними умовами

$$
\left.\frac{\partial \widetilde{\theta}_{n}^{(i)}}{\partial r}\right|_{r=\sqrt{R^{2}-(z-R)^{2}}}=\widetilde{G}_{n}^{(i)}(z),\left.\quad \frac{\partial \widetilde{\theta}_{n}^{(i)}}{\partial z}\right|_{z=R}=\widetilde{\Lambda}_{n}^{(i)}(r)
$$


«Системні технологіï» 5 (136) 2021 «System technologies»

де $\widetilde{G}_{n}^{(i)}(z)=G_{n}^{(i)}(z)\left(1+\frac{1}{s \tau_{r}}\right) ; \quad \widetilde{\Lambda}_{n}^{(i)}(z)=\Lambda_{n}^{(i)}(z)\left(1+\frac{1}{s \tau_{r}}\right)$.

Для розв’язання крайової задачі (9)-(10) застосовуємо інтегральне перетворення:

$$
\bar{f}\left(\mu_{n, k}\right)=\iint_{\mathrm{D}} Q\left(\mu_{n, k}, r, z\right) \cdot r \cdot \mathrm{f}(r, z) d \sigma .
$$

Власні функції $Q\left(\mu_{n, k}, r, z\right)$ і власні значення $\mu_{n, k}$ знаходяться із розв’язку спектральної задачі:

$$
\begin{gathered}
\frac{\partial^{2} Q}{\partial r^{2}}+\frac{1}{r} \frac{\partial Q}{\partial r}-\frac{n^{2}}{r^{2}} Q+\frac{\partial^{2} Q}{\partial z^{2}}+\mu_{n, k} \cdot Q=0 \\
\left.\frac{\partial Q}{\partial r}\right|_{r=\sqrt{R^{2}-(z-R)^{2}}}=0,\left.\quad \frac{\partial Q}{\partial z}\right|_{z=R}=0 .
\end{gathered}
$$

Власні функції $Q\left(\mu_{n, k}, \rho, z\right)$ і власні значення $\mu_{n, k}$ в (12)- (13) находяться по формулам, які приведені в [8], а формула оберненого перетворення має вигляд:

$$
\mathrm{f}(\rho, z)=\sum_{j=1}^{\infty} \frac{Q\left(\mu_{n, k}, r, z\right)}{\left\|Q\left(\mu_{n, k}, r, z\right)\right\|^{2}} \bar{f}\left(\mu_{n, k}\right) .
$$

Застосовуємо до системи диференціальних рівнянь (9) інтегральне перетворення (11) в результаті одержуємо систему алгебраїчних рівнянь відносно $\widetilde{\widetilde{\theta}}_{n}^{(i)}$ :

$$
\begin{aligned}
& s \overline{\widetilde{\theta}}_{n}^{(i)}+\vartheta_{n}^{(i)}\left(\overline{\widetilde{\theta}}_{n}^{\left(m_{i}\right)}+\tau_{r} s \overline{\widetilde{\theta}}_{n}^{\left(m_{i}\right)}\right)+\tau_{r} s^{2} \overline{\widetilde{\theta}}_{n}^{(i)}=q_{n, k}\left(\frac{\widetilde{\Omega}_{\mathrm{n}, \mathrm{k}}^{(\mathrm{i})}}{\mu^{2}{ }_{n, k}}-\overline{\widetilde{\theta}}_{n}^{(i)}\right) \\
& \text { де } \widetilde{\Omega}_{\mathrm{n}, \mathrm{k}}^{(\mathrm{i})}=\underset{0}{h} \underset{0}{h} Q\left(\mu_{n}, \varsigma(z), z\right) \widetilde{G}_{n}^{(i)}(z) d z+\oint_{L} r\left(Q\left(\mu_{n, k}, r, z\right) \frac{\partial \widetilde{\theta}_{n}^{(i)}}{\partial z}-\widetilde{\theta}_{n}^{(i)} \frac{\partial Q\left(\mu_{n, k}, r, z\right)}{\partial z}\right) d r ; \\
& q_{n, k}=\mathrm{a} \mu_{n, k}^{2} .
\end{aligned}
$$

Криволінійний інтеграл обчислюється по замкненому додатно орієнтованому контуру (рис.2): 
«Системні технологіï» 5 (136) 2021 «System technologies»

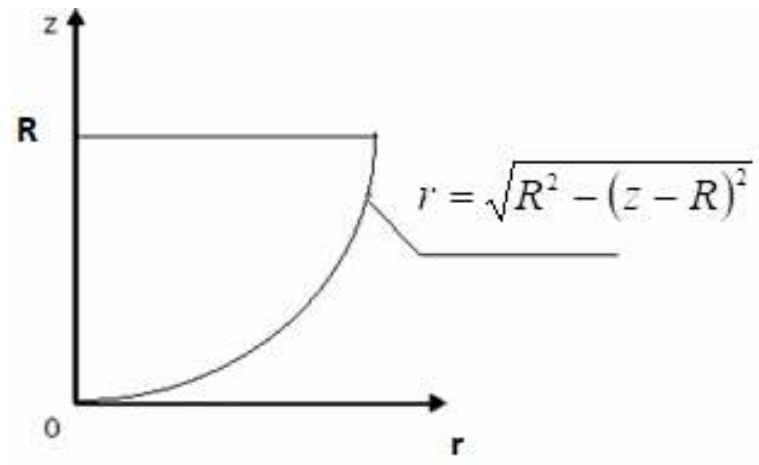

Рисунок 2 - Замкнутий контур із твірною лінією $r=\sqrt{R^{2}-(z-R)^{2}}$

Розв’язавши систему рівнянь (15), одержуємо

$$
\overline{\widetilde{\theta}}_{n}^{(i)}=a \cdot \frac{\widetilde{\Omega}_{\mathrm{n}, \mathrm{k}}^{(\mathrm{i})}\left(\tau_{r} s^{2}+s+\mathrm{q}_{\mathrm{n}, \mathrm{k}}\right)+(-1)^{i+1} \omega n \widetilde{\Omega}_{\mathrm{n}, \mathrm{k}}^{\left(m_{i}\right)}\left(1+s \tau_{r}\right)}{\left(\tau_{r} s^{2}+s+\mathrm{q}_{\mathrm{n}, \mathrm{k}}\right)^{2}+\omega^{2} n^{2}\left(1+s \tau_{r}\right)^{2}} .
$$

Застосовуючи до зображення функцій (16) формули оберненого перетворення Лапласа (7), одержуємо оригінали функцій:

$$
\begin{aligned}
& \left.\bar{\theta}_{n}^{(1)}\left(\mu_{n, k}, t\right)=\sum_{j=1}^{2} \zeta_{n, k}\left(s_{j}\right)\right)\left(\widetilde{\Omega}_{\mathrm{n}, \mathrm{k}}^{(1)}\left(s_{j}\right)\left[\left(2 \tau_{r} s_{j}+1\right)+\tau_{r} \omega n \mathrm{i}\right]+\widetilde{\Omega}_{\mathrm{n}, \mathrm{k}}^{(2)}\left(s_{j}\right)\left[\tau_{r} \omega n-\left(2 \tau_{r} s_{j}+1\right) \mathrm{i}\right]\right\} \\
& \left.\left.\left(e^{s_{j} t}-1\right)+\sum_{j=3}^{4} \zeta_{n, k}\left(s_{j}\right)\right) \widetilde{\Omega}_{\mathrm{n}, \mathrm{k}}^{(1)}\left(s_{j}\right) \cdot\left[\left(2 \tau_{r} s_{j}+1\right)-\tau_{r} \omega n \mathrm{i}\right]+\widetilde{\Omega}_{\mathrm{n}, \mathrm{k}}^{(2)}\left(s_{j}\right)\left[\tau_{r} \omega n+\left(2 \tau_{r} s_{j}+1\right) \mathrm{i}\right]\right\} . \\
& \cdot\left(e^{s_{j} t}-1\right),
\end{aligned}
$$

$\left.\bar{\theta}_{n}^{(2)}\left(\mu_{n, k}, t\right)=\sum_{j=1}^{2} \zeta_{n, k}\left(s_{j}\right)\right)\left\{\widetilde{\Omega}_{\mathrm{n}, \mathrm{k}}^{(2)}\left(s_{j}\right)\left[\left(2 \tau_{r} s_{j}+1\right)+\tau_{r} \omega n \mathrm{i}\right]-\widetilde{\Omega}_{\mathrm{n}, \mathrm{k}}^{(1)}\left(s_{j}\right)\left[\tau_{r} \omega n-\left(2 \tau_{r} s_{j}+1\right) \mathrm{i}\right]\right\}$

$\left(e^{s_{j} t}-1\right)+\sum_{j=3}^{4} \zeta_{n, k}\left(s_{j}\right)\left(\widetilde{\Omega}_{\mathrm{n}, \mathrm{k}}^{(2)}\left(s_{j}\right)\left[\left(2 \tau_{r} s_{j}+1\right)-\tau_{r} \omega n \mathrm{i}\right]-\widetilde{\Omega}_{\mathrm{n}, \mathrm{k}}^{(1)}\left(s_{j}\right)\left[\tau_{r} \omega n+\left(2 \tau_{r} s_{j}+1\right) \mathrm{i}\right]\right\}$.

$\left(e^{S_{j} t}-1\right)$

де $\zeta_{n, k}\left(s_{j}\right)=\frac{0.5 s_{j}^{-1} a}{\left(2 \tau_{r} s_{j}+1\right)^{2}+\left(\tau_{r} \omega n\right)^{2}}$, а значення $s_{j}$ для $\mathrm{j}=1,2,3,4$ визначаються

за формулами

$$
s_{1,2}=\frac{\left(\tau_{r} \omega n i-1\right) \pm \sqrt{\left(1+\tau_{r} \omega n i\right)^{2}-4 \tau_{r} q_{n, k}}}{2 \tau_{r}}, s_{3,4}=\frac{\left(\tau_{r} \omega n i+1\right) \pm \sqrt{\left(1-\tau_{r} \omega n i\right)^{2}-4 \tau_{r} q_{n, k}}}{2 \tau_{r}} .
$$

Таким чином з урахуванням формул обернених перетворень (5) і (14) одержує164 
«Системні технології» 5 (136) 2021 «System technologies»

мо температурне поле тіла, що обертається з постійною кутовою швидкістю $\omega$ навколо осі OZ, із урахуванням кінцевої швидкості поширення тепла:

$$
\theta(r, \varphi, z, t)=\sum_{n=-\infty}^{+\infty}\left\{\sum_{\mathrm{k}=1}^{\infty}\left[\bar{\theta}_{n}^{(1)}\left(\mu_{n, k}, t\right)+\mathrm{i} \bar{\theta}_{n}^{(2)}\left(\mu_{n, k}, t\right)\right] \frac{Q\left(r, z, \mu_{n, k}\right)}{\left\|Q\left(r, z, \mu_{n, k}\right)\right\|^{2}}\right\} \exp (\operatorname{in} \varphi)
$$

де $\bar{\theta}_{n}^{(1)}\left(\mu_{n, k}, t\right), \bar{\theta}_{n}^{(2)}\left(\mu_{n, k}, t\right)$ визначаються за формулами (17), (18).

Висновки. Вперше побудована математична модель розрахунку температурних полів для півсферичного тіла , яке наближено моделює роботу алмазного долота, з урахуванням кутової швидкості буріння та кінцевої швидкості поширення тепла у вигляді крайової задачі математичної фізики для гіперболічного рівняння теплопровідності з граничними умовами Неймана.

В роботі побудоване нове інтегральне перетворення для двовимірного кінцевого простору, із застосуванням якого знайдено температурне поле у вигляді збіжного ряду.

Знайдений розв’язок може знайти застосування для прогнозування можливої величини механічної швидкості алмазного буріння, сприяти правильному вибору технологічних параметрів, об'єктивного контролю їх в процесі буріння, дозволяє намітити шляхи вдосконалення роботи алмазного долота.

\section{ЛІТЕРАТУРА / ЛИТЕРАТУРА}

1. Александров В. А. Температурное поле и износ неоднородного алмазного круга при конвективном теплообмене / В.А.Александров, А.Н. Жуковский, В. А. Мечник // Трение и износ. - 1994. - Т. 15, - № 1. - С. 27 - 35.

2. Бондаренко Н.А. Температурное состояние алмазных буровых долот/ Н.А.Бондаренко, А.Н. Жуковский, В.А. Мечник // Доповіді НАН України. - 2006. - № 10. - С. 95 - 102.

3. Кожевников А.А. Математическая модель температурного режима алмазной коронки при бурении скважины / А.А. Кожевников, А.Ю. Дреус, И.И. Мартыненко, Ю.А. Бакаржиев // Матеріали міжнар. конф. “Форум гірників-2005”.Дніпропетровськ: НДУ, 2005. - Т. 2. - С. 250-257.

4. Горшков Л.К. Температурные режимы алмазного бурения/Л.К. Горшков, В.Г. Гореликов. - М.: Недра, 1992. - 212 с.

5. Бердник М.Г. Математичне моделювання температурного поля в циліндрі, який обертається, з урахуванням кінцевої швидкості поширення тепла / М.Г. Бердник // Питання прикладної математики і математичного моделювання.Дніпропетровськ: ДНУ, 2005.-С. 37-44. 


\section{«Системні технології» 5 (136) 2021 «System technologies»}

6. Маркович Б. М. Рівняння математичної фізики/Б. М. Маркович. - Львів: Львівська політехніка, 2010. - 384 с.

7. Лопушанська Г.П. Перетворення Фур’є, Лапласа: узагальнення та застосування/ Г.П. Лопушанська, А.О. Лопушанський, О.М. М’яус. - Львів: ЛНУ ім. Івана Франка, 2014. -152 с.

8. Berdnyk M. The mathematic model and method for solving the dirichlet heatexchange problem for empty isotropic rotary body / M. Berdnyk // Non-Traditional Technologies in the Mining Industry. Solid State Phenomena Vol. 277 - Trans Tech Publications, Switzerland- 2018 - pp 168-177.

\section{REFERENCES}

1. Aleksandrov V. A. Temperaturnoe pole i iznos neodnorodnogo almaznogo kruga pri konvektivnom teploobmene / V.A.Aleksandrov, A.N. Zhukovskij, V. A. Mechnik // Trenie i iznos. - 1994. - T. 15, - № 1. - S. 27 - 35.

2. Bondarenko N.A. Temperaturnoe sostojanie almaznyh burovyh dolot/ N.A.Bondarenko, A.N. Zhukovskij, V.A.Mechnik, // Dopovidi NAN Ukraïni. - 2006. № 10. - S. 95 - 102.

3. Kozhevnikov A.A. Matematicheskaja model' temperaturnogo rezhima almaznoj koronki pri burenii skvazhiny / A.A. Kozhevnikov, A.Ju. Dreus, I.I. Martynenko, Ju.A. Bakarzhiev // Materiali mizhnar. konf. “Forum girnikiv-2005”.-Dnipropetrovs'k: NDU, 2005. - T. 2. -S. 250-257.

4. Gorshkov L.K. Temperaturnye rezhimy almaznogo burenija/L.K. Gorshkov, V.G. Gorelikov. - M.: Nedra, 1992. - 212 s.

5. Berdnik M.G. Matematichne modeljuvannja temperaturnogo polja v cilindri, jakij obertaєt'sja, z urahuvannjam kincevoï shvidkosti poshirennja tepla / M.G. Berdnik // Pitannja prikladnoï matematiki i matematichnogo modeljuvannja. Dnipropetrovs'k: DNU, 2005. - S. 37-44.

6. Markovich B. M. Rivnjannja matematichnoï fiziki/B. M. Markovich. - L'viv: L'vivs'ka politehnika, 2010. - 384 c.

7. Lopushanska, G.P. Peretvorennja Furje, Laplasa: uzagalnennja ta zastosuvannja. Lviv.: LNU im. Ivana Franka, 2014. -152 s.

8.Berdnyk M. The mathematic model and method for solving the dirichlet heatexchange problem for empty isotropic rotary body / M. Berdnyk // Non-Traditional Technologies in the Mining Industry. Solid State Phenomena Vol. 277 - Trans Tech Publications, Switzerland- 2018 - pp 168-177. 


\section{«Системні технології» 5 (136) 2021 «System technologies»}

\section{Математическая модель и метод решения обобщенной задачи Неймана теплообмена тела полусферической формы}

Построена математическая модель расчета температурных полей в вращающемся полусферическом теле, с учетом конечной скорости распространения тепла, в виде краевой задачи математической физики для гиперболического уравнения теплопроводности. Построенное интегральных преобразования для двумерного конечного пространства, с применением которого найдено температурное поле в виде сходящихся рядов по функциям Фурье.

\section{Mathematical model and method for solving the generalized neumann problem of heat exchange of a hemispherical body}

Currently, the issue of the distribution of temperature fields in the workpieces under the new method of heating, which is carried out by rotating the blanks in the magnetic field of a direct current, which is created in exciters with superconducting windings without knowledge of which it is impossible to implement its technical implementation with high technical and economic indicators, is not sufficiently studied. In this case, a small number of scientific works is devoted to the study of electromagnetic and thermal phenomena within the workpiece during its rotation in a magnetic field of direct current. Therefore, the task of developing mathematical methods for modeling the temperature fields in the workpieces with the induction heating of the metal with the use of innovative technology, the solution of which is dedicated to this work, is very relevant.

The purpose of the article is to construct a new generalized spatial mathematical model for calculating temperature fields in the workpieces in the form of a paraboloid of rotation, rotating with a constant angular velocity, taking into account the final rate of heat propagation in the form of the boundary value problem of mathematical physics, as well as finding solutions to the resulting boundary value problem.

For the first time, a mathematical model for calculating the temperature fields in a paraboloid of rotation has been constructed, taking into account the finite rate of propagation of the rotating heat in the form of a boundary value problem of mathematical physics for hyperbolic heat equations with boundary conditions of Neumann. An integral transformation for a two-dimensional finite space was constructed, with the use of which a temperature field was found in the form of convergent series in Fourier functions.

The solution of the generalized boundary value problem of heat exchange of the rotational paraboloid, which is rotated, taking into account the finiteness of the velocity of heat propagation, can be found in the modulation of temperature fields that arise during induction heating, which is carried out by rotating the blanks in a magnetic field of direct current created in the exciters with superconducting windings. 
Бердник Михайло Геннадійович - кандидат фізико-математичних наук, доцент, Національний технічний університет "Дніпровська політехніка", доцент кафедри програмного забезпечення комп’ютерних систем, м.Дніпро, Україна. Алексєєв Олексій Михайлович - кандидат технічних наук, доцент, Національний технічний університет "Дніпровська політехніка", доцент кафедри програмного забезпечення комп’ютерних систем.

Бердник Михаил Геннадьевич - кандидат физико-математических наук, доцент, Национальный технический университет "Днепровская политехника", доцент кафедры программного обеспечения компьютерных систем, г.Днепр, Украина.

Алексеев Алексей Михайлович - кандидат технических наук, доцент, Национальный технический университет "Днепровская политехника", доцент кафедры программного обеспечения компьютерных систем.

Berdnyk Mykhailo Hennadiiovych - Candidate of Physics and Mathematics, assistant professor, National Technical University Dnipro Polytechnic, Senior Lecturer of computer software systems, Dnipro, Ukraine.

Aleksieiev Oleksii Mykhailovich - Candidate of Technical Sciences, assistant professor, National Technical University Dnipro Polytechnic, Senior Lecturer of computer software systems. 